\title{
Cooperative Data Management in Wireless Sensor Networks
}

\author{
Hajar Mousannif \\ FSTG, Cadi Ayyad University, B.P.: 549 Av. Abdelkarim Elkhattabi, Guéliz, Marrakech, Morocco \\ E-mail: hajar.mousannif@gmail.com \\ www.ucam.ac.ma \\ Ismail Khalil \\ Department of telecooperation, Johannes Kepler University, Altenberger Strasse 69, A-4040 Linz, Austria \\ E-mail: ismail.khalil@jku.at \\ www.jku.at \\ Received 24 November 2011 \\ Accepted 24 February 2012
}

\begin{abstract}
Introducing cooperation into a wireless sensor network (WSN) has gained much attention in the recent few years mainly because of the significant effect it has on optimizing energy consumption and on enhancing the lifetime and the overall performance of the network. Cooperation can be exploited at different levels, ranging from a collection of nodes collaborating to forward the data they gathered from the environment towards the base station through efficient data aggregation and clustering techniques, to nodes collaborating to report events occurrences, track targets or control the topology. Motivated by a large variety of attractive wireless sensor applications, such as environmental monitoring, smart environments and healthcare applications, we survey mechanisms that take advantage of cooperation among sensor nodes in the network for the purpose of delivering information reliably and efficiently to nodes of the network that are interested in receiving it. We provide detailed overviews and highlight the importance of cooperation from different perspectives.
\end{abstract}

Keywords: WSN, Cooperation, data management, data aggregation, topology control, survey

\section{Introduction}

A wireless sensor network is a collection of a large number of small, self-powered and inexpensive devices organized into a cooperative network. Individual sensor nodes are connected to other nodes in their vicinity through a wireless network, and use a multi-hop routing protocol to communicate with nodes that are spatially distant. Depending on the application, the onboard sensors collect acoustic, seismic, infrared or magnetic information about the environment, using either continuous or event driven working modes. In wireless sensor networks, one or more base stations operating as data sinks and/or as gateways to other networks like Internet are also present to subscribe specific data streams by expressing interest or queries. The sensors in the network act as sources, which detect environmental events, such as fire, flooding, chemical leak or air condition change and push the sensed data to the appropriate sink. ${ }^{1}$ Thus, wireless sensor networks have emerged as a new information-gathering (or eventdetecting) paradigm relying on the collective and cooperative effort of numerous sensor nodes.

Cooperation in a wireless sensor network refers to the interaction between different entities in the network for the purpose of delivering information reliably and 
efficiently to the destination. In fact, nodes can help each others' data forwarding so that all parties involved in such cooperation benefit from this collaborative effort (i.e., energy consumption for packet relaying reduces and the lifetime of the network is prolonged). Energy is the most serious limitation in a wireless sensor network. Energetic resources are limited and battery replacement is not an option for networks with thousands of physically embedded sensor nodes. Each sensor in the network consumes power, not only in sensing data, but also for processing the data and transmitting these processed information for further routing. The most energy-consuming component is the $\mathrm{R} / \mathrm{F}$ module that provides wireless communications. It has been widely argued that communication of data over wireless links consumes much more energy than sensing and data processing. ${ }^{2}$

In this paper, we survey mechanisms which utilize nodes' cooperation for data dissemination in a wireless sensor networks. We classify these mechanisms into four categories: $1^{\circ}$ ) mechanisms using cooperation for data gathering, $2^{\circ}$ ) mechanisms using cooperation for event detection and target tracking, $3^{\circ}$ ) mechanisms using cooperation for topology control and $4^{\circ}$ ) mechanisms using cooperation for prolonging the network lifetime. We study several important performance properties of each approach and highlight the importance of cooperation among sensor nodes from different perspectives.

The remainder of this paper will be organized as follows: Section 2 surveys mechanisms that exploit cooperation for data gathering including data aggregation, data correlation and data co-occurrence. In Section 3, we overview mechanisms that use nodes' cooperation to detect events and track targets. We continue in section 4 with techniques that take advantage of cooperation among sensor nodes to control the topology. We finish in Section 5 by presenting specific mechanisms that aim at prolonging the network lifetime through cooperation. Section 6 concludes our work.

\section{Cooperation for data gathering}

A common activity of many applications of WSN is that of data gathering of sensed data at a distant base station (BS). Such applications include environmental monitoring (See Refs. 3 and 4), habitat monitoring ${ }^{5}$ and structure health monitoring ${ }^{6}$. An important challenge associated with data gathering is to reduce the message cost to minimize the bandwidth usage of the network and the energy consumption of the sensor nodes. Data aggregation and in-network processing techniques have been investigated as efficient approaches to achieve significant energy savings in WSN by combining data arriving from different sensor nodes at some aggregation points, eliminating redundancy, and minimizing the number of transmission before forwarding data to the sinks. In specific applications, however, the base station is interested in getting every measurement from all the sensors rather than the aggregated data, in order to determine the status of the observing field and make appropriate decisions. In these cases, Data correlation and data co-occurrence have proved to achieve high energy efficiency.

\subsection{Data aggregation}

In many applications of WSN (e.g., military battlefields, target field imaging, intrusion detection, surveillance, and inventory control), data collected by many sensors is based on common phenomena, and hence there is a high probability that this data has some redundancy (or correlation). Data aggregation ${ }^{7}$ has been investigated recently as an efficient approach to achieve significant energy savings in WSN by combining data arriving from different sensor nodes at different aggregation points, eliminating redundancy, and minimizing the number of transmission before forwarding data to the sinks. A number of studies that compared aggregation schemes, e.g., authors in Refs. 8 and 9 concluded that enhanced network throughput and more potential energy savings are highly possible using data aggregation.

Data aggregation combines data from different sources by using functions such as suppression (eliminating duplicates), minimum, maximum, average (see Fig. 1. as an example), variance, approximate histograms, uniform fixed-size samples, measurements of high frequency, and various sketches such as those in Refs. 10-12. Some of these functions can be performed either partially or fully in each sensor node, by allowing sensor nodes to conduct in-network data reduction. In some network architectures, all aggregation functions are assigned to more powerful and specialized nodes. Data aggregation is also feasible through signal processing techniques. In that case, it is referred as data fusion. 
The general data aggregation problem can be defined as: given $\mathrm{m}$ sources and one sink in a network composed of $\mathrm{n}$ nodes $(\mathrm{m}<\mathrm{n})$, find a minimum weight sub-graph that includes all sources. This is a well known NP-complete problem, known as the Steiner Tree Problem (STP). ${ }^{13}$ There are many approximation algorithms for solving STP. ${ }^{14}$ From sensor networks' perspective, particularly, for many monitoring applications with a periodic collection pattern, a treebased topology (such as in Refs. 15 and 16) was often adopted because of its simplicity and power efficiency.

\subsection{Data correlation and data co-occurrence}

In this section, we focus on applications in which each sensor continuously monitors a field of interest, and the base station is interested in getting every measurement from all the sensors, in order to determine the status of the observing field and make appropriate decisions.

As pointed in the previous section, data aggregation provides a great opportunity for reducing the communication overhead by fusing several packets from different sensors into one packet to eliminate the redundant transmissions, using the summary data (e.g., SUM) and/or exemplary data (e.g., MIN and MAX). But, since a single aggregated value represents the overall sensing field, it may be insufficient for analyzing the correlation among sub-regions of the sensor field. Furthermore, in a large sensor network, sensor readings may not accurately reflect the current state of the network due to the device noise, network failure and so on. Thus, in many cases, the users are interested in individual readings of sensors, rather than aggregated data. However, if each sensor transmits its every measurement to the sink, this will lead to high energy inefficiency since often there is redundancy and/or dependency among the sensor measurements. How to identify the data redundancy/dependency and utilize them on improving energy efficiency of data gathering has been one of the attractive topics.

In fact, two techniques are identified in the literature for improving energy efficiency of data gathering: Data correlation and data co-occurrence. There are two major differences between the two ${ }^{17}$ : The first is that correlation attempts to capture monotonicity trends (e.g. linear dependencies) between sequences while cooccurrence does not provide information about such monotonicity trends; instead, it attempts to quantify the

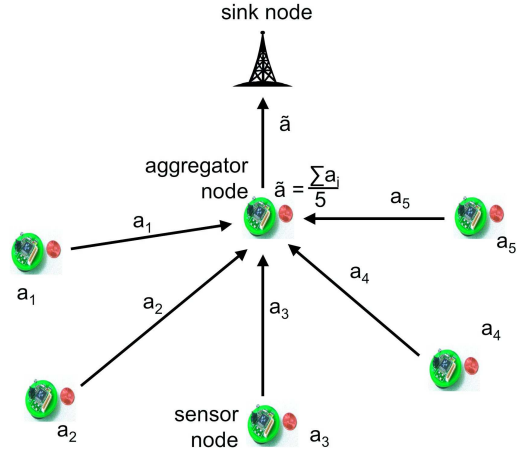

Fig. 1. Illustration of data aggregation

trend that two values tend to occur simultaneously (e.g. non-linear dependencies), and is capable of handling discrete enumerated data. Two measurements co-occur if the set of times at which they are measured are similar. The second difference is that data co-occurrence can appear in both densely and sparsely deployed sensor networks while data correlation is normally expected only in densely deployed sensor networks. Authors in Ref. 17 present a cooperative data gathering protocol exploiting measurements co-occurrence. The protocol allows sensors to discover co-occurring elements and to collaborate by sharing the load of communicating such co-occurring elements by informing the base station and then taking turns in communicating those measurements to the base station. It also allows the base station to make inferences about the sensor measurements.

In a sensor field, two correlations among sensor readings can be distinguished ${ }^{18}$ :

- Temporal correlation where the change pattern of future sensor readings is equal to or similar to that of previous sensor readings in a sensor,

- Spatial correlation where the change patterns of sensor readings of the adjacent sensors are the same or similar. For example, consider the query: what is the temperature in the region defined by the rectangle (x1, y1, x2, y2)? Given the typical dense deployments of sensors in WSN, it is likely that the sensing regions of two different sensors within the rectangular region overlap. Consequently, the data reported by these sensors are spatially correlated.

Authors in Ref. 18 use both types of correlations to reduce the communication overhead in their proposed approximate data gathering technique. In their approach, close sensors are grouped spatially as a cluster. In a cluster, each member of a cluster reports its approximate sensor reading to the cluster head. A 
cluster head also computes an estimated reading for its own reading. If the estimated reading is outside the error bound, the cluster header sends its reading to the base station otherwise it sends the member's actual reading to the base station. The authors also devised a localized mechanism, using the spatial correlation, in which nodes cooperate to distribute energy among them by allowing all or certain portions of a cluster header's children to migrate to other nodes to avoid that a sensor acts as a cluster head permanently.

\subsection{Cooperative data aggregation}

The Cooperative communication is a new research area, in which one or several nodes cooperate with a sender to transmit a data packet to the next-hop node along a path to achieve network energy saving and throughput increasing.

The data aggregation methods early presented in section 2.1 usually took the short-range transmissions instead of long-range transmissions so as to decrease the power cost of wireless communication. Several works, however, studied the energy-efficient communication model based on cooperative transmission for wireless sensor networks. These solutions analyzed the minimum transmission power under the different relay selection and various communication paradigms, including endto-end communication, broadcast communication and cooperative communication, etc.

In Ref. 19, authors have defined the Cooperative data aggregation (CDA) problem as how to construct a topology and select a set of cooperative node pairs for data aggregation, so that all the sensor nodes can finally send the sensed data or fused data to sink node with direct transmission or cooperative transmission schemes. The target of this problem is to minimize the total power consumption of data aggregation. The authors argued convincingly that cooperative communication technology can be incorporated into data aggregation for the sake of energy efficiency and lifetime extension. They illustrated their point of view through a network example (as depicted in Fig. 2.). They found that under the conventional manner, the minimum total power consumption is 60 for data aggregation, illustrated by Fig. 2. (b). With the cooperative communication, nodes $\mathrm{v} 1$ and $\mathrm{v} 2$ first exchange their sensed data, which costs the power of 20. Then, two nodes compute a consensus fused result based on the received data, and cooperatively transmit
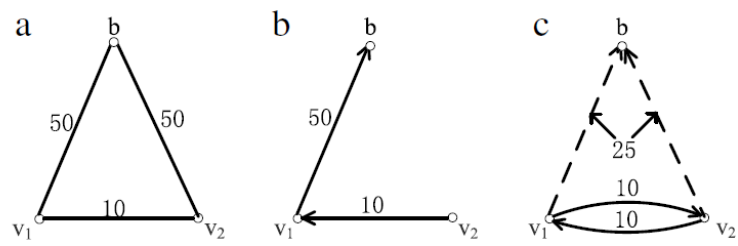

Fig. 2. Illustration of cooperative data aggregation ${ }^{19}$

the result to base station $b$. It costs the power costs of 25. As a result, the total power consumption of data aggregation is only 45 instead of 60 under the conventional manner. Obviously, power saving is obtained.

Authors in Ref. 20 propose a cooperative relay selection scheme in which each node on the route selects the optimum cooperative node from all the potential cooperative nodes to construct the cooperative link with maximum throughput so that cooperative route with maximum network throughput from source to destination can be set up. In their selection scheme, all the nodes in the network are divided into several onehop clusters. Local routing information is exchanged between neighboring nodes to establish routes between cluster heads (CHs) in adjacent clusters, which are further used to propagate to nonadjacent clusters to set up routes between them. Their simulation results showed that an improvement of network throughput by up to $30 \%$ in comparison to a non-cooperative approach.

\section{Cooperation for event detection and target tracking}

An event can be defined as an exceptional change in the environment parameters such as temperature, light, humidity, etc. To reduce energy consumption, sensors operate in a silent monitoring state and are " "programmed" to notify about events. The event-driven model of sensor networks supports many applications, such as flood detection, ${ }^{21}$ telemonitoring of human health status, ${ }^{22}$ vehicle anti-theft ${ }^{23}$ and target tracking. ${ }^{24}$ Among these applications, those that involve mobile events are more challenging than that for static events, since mobile events need to be tracked in a continuous manner. Target tracking is usually used for monitoring a geographic region where mobile persons or vehicles may intrude.

Some applications have the requirement of timely delivery. For example, sensor and actor networks ${ }^{25}$ 
require sensors to collect and propagate information in a timely manner so that actors can take timely actions. When a target enters an area of interest, the delay to report the sensed event could be critical. If the reported event is not received by the sink node within the deadline, the end-to-end (ETE) delay requirement is not satisfied

Distributed event detection in WSN is the process of observing and evaluating an event using multiple sensor nodes, in which the global decision is made by fusing local decisions from nodes to increase event detection accuracy and reduce communication overhead and risk of network node failures. ${ }^{26}$ Event detection is a critical issue in wireless sensor networks. According to the event characteristics, the occurrence of the event should be quickly and reliably conveyed to the sink node for decision making. However with the characteristics of low power, low cost, and short communication range, a sensor node has to cooperate with other nodes in order to report the event occurrence to the base station.

In Ref. 26, authors propose a collaborative event detection scheme for clustered WSN that utilizes fuzzy logic to evaluate the credibility of clusters. Sensor nodes will send local decisions to their corresponding Cluster head initially responsible for the surveillance of a subregion of the whole region of interest. The Cluster head will make decision about event presence/absence in the sub-region based on the local decisions received from sensors. The decisions from all cluster heads will then be transmitted to the fusion center along with their detection performance. At the fusion center, instead of treating all clusters equally, the credibility of each cluster is taken into account when the fusion center makes the final decision. The cluster with higher detection probability and lower false alarm probability will be given greater credibility.

Authors in Ref. 27 proposed a fully distributed and collaborative protocol, to event detection and tracking in wireless heterogeneous sensor networks (WHSNs), which consists of various types of sensor nodes with different sensing units. Their protocol mainly adopts the collaborations of the same and the different types of sensor nodes to determine the urgent and to select the border sensor nodes for event detection and tracking. Authors first tackled the event boundary determination issue in critical scenarios such as fire or pollution by a hazardous gas, where the event is compound of multiple attributes and may occupy a large area and tend to increase or decrease in size, or change in shape with time elapsed. They further proposed a dynamic role assignment that relies on the cooperation of the same and the different types of sensor nodes so that the event can be tracked.

In Ref. 28, authors analyzed the impact of the number of selected reporting nodes on WSN performance (i.e. energy consumption and reporting time). They proved that using only a small number of sensor nodes to report the event occurrence rather than all the nodes in the event area reduces considerably the energy consumption and improves the network lifetime. They also showed that when only one reporting node is activated, the maximal network lifetime is achieved. The appropriate reporting node(s) is/are selected through nodes cooperation.

\section{Cooperation for topology control}

The goal of topology control is to dynamically tune the nodes' transmission power to enforce specific properties of the communication graph, while ensuring that data can be forwarded efficiently to the sink. ${ }^{29}$ If a node powers down its radio, it is essentially disconnected from the rest of the network topology and therefore can no longer perform packet relaying. The sensors and processor, however, can still be active, as they are much less power hungry. As density increases, more nodes can be sleeping, resulting in further energy savings. Topology control contributes to power saving mainly in two ways in sensor networks ${ }^{30}$ :

- It allows non-routing nodes (or "sensing nodes") to maintain lower duty cycle because they don't have to receive packets for the routing purpose. This serves to reduce idle listening and overhearing since sensing nodes can simply turn off their radio most times while performing sensing

- $\quad$ Routing nodes can act as data aggregation points as all the packets are forwarded through these nodes. This serves to reduce the amount of traffic on the routing backbone.

Most protocols for topology control in WSN leverage the concepts of cooperation among sensors to ensure global network connectivity properties. Two approaches are identified in the literature to ensure such properties. In the first approach (like in Refs. 31 and 32), a hierarchy of Cluster head nodes is obtained to construct a connected communication backbone of the network. The idea is to designate only a subset of nodes to carry out the network control functions and thus to reduce the 
communication overhead. The aim of the second (such as in Refs. 33 and 34) is to control the number of neighboring node/cluster heads, as the physical degree of a node strongly influences the utilization of available bandwidth and energy consumption.

\subsection{Clustering}

Since large population of sensors (hundreds or even thousands) are expected in most WSN applications, designing and operating such large size network would require scalable architectural and management strategies. Grouping sensor nodes into clusters has been widely pursued by the research community in order to achieve the network scalability objective. Every cluster would have a leader, often referred to as the Cluster head $(\mathrm{CH})$. Every cluster head is responsible for a group of child nodes in the cluster (Fig. 3.). When cluster heads receive queries from the aggregate nodes, they send the queries to the child nodes to get results and, in return, send back results to the aggregate nodes (AN). ${ }^{35}$ A CH may be elected by the sensors in a cluster or preassigned by the network designer. A CH may also be just one of the sensors or a node that is richer in resources. The cluster membership may be fixed or variable. Many clustering algorithms have been proposed in the literature. An interesting survey on clustering algorithms for wireless sensor networks can be found in Ref. 36. These proposed clustering techniques widely vary depending on the node deployment and bootstrapping schemes, the pursued network architecture, the characteristics of the $\mathrm{CH}$ nodes and the network operation model.

The LEACH (low-energy adaptive clustering hierarchy) protocol, ${ }^{37}$ for example, selects cluster heads periodically and drains energy uniformly by role rotation. Each node decides itself whether or not a Cluster head distributed by a probability. In PEGASIS (power efficient gathering in the sensor information systems), ${ }^{38}$ nodes will be organized to form a chain using a greedy algorithm so that each node transmits to and receives from one of its neighbors. A randomly selected node from the chain will forward the aggregated data to the base station, thereby reducing per round energy expenditure compared to LEACH. The requirement of global knowledge of the network topology makes this method difficult to implement. HEED (Hybrid, Energy-Efficient, Distributed Clustering $^{39}$ is a distributed clustering algorithm,

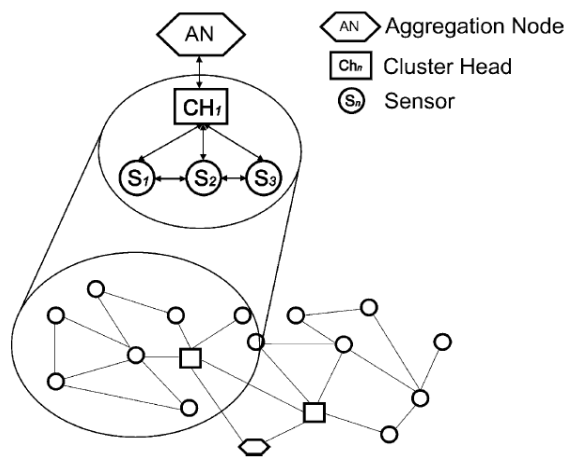

Fig. 3. Clustering in WSN

which selects the cluster heads stochastically. HEED uses a hybrid criterion for cluster head selection, which considers the residual energy of the node and a secondary parameter, such as the node's proximity to its neighbors or the node's neighbor degree. HEED prolongs the network lifetime by ensuring balanced energy dissipation as well as uniform distribution of cluster head nodes in network scenarios that contain uniformly dispersed sensor nodes.

Clustering has many advantages. It can stabilize the network topology at the level of sensors and thus cuts on topology maintenance overhead. Sensor would only insure their connections to the CHs. Clustering can also enhance the network operation and prolong the network lifetime.$^{40} \mathrm{~A} \mathrm{CH}$ can schedule activities in a round-robin order in the cluster so that nodes can switch to the lowpower sleep mode most of the time and reduce their energy consumption. The cluster head role can also be rotated in order to distribute the energy consumption evenly among all sensors in the network. Moreover, a $\mathrm{CH}$ can also aggregate the data collected by the sensors in its cluster and thus decrease the number of relayed packets.

\subsection{Neighborhood-based topology control}

Within this field, neighborhood-based topology control solutions aim at maintaining a given local degree at each node, by dynamically adjusting the node's transmission power to control the number of neighboring node. Approaches in Refs. 41-44, let each node arbitrarily increase its transmission power until $\mathrm{K}$ neighbors are heard. Such approaches usually resort to maximum power transmissions during the discovery phase to detect all the nodes potentially reachable. Once this information is acquired, each node tunes its 
transmission range (or power) to achieve the desired neighbor degree. Since transmitting at maximum power is likely to create interference among node transmissions thus preventing some nodes to correctly receive packets from other nodes, some solutions propose that each node starts transmitting at low power and incrementally increases until $\mathrm{K}$ neighbors are contacted. Authors in Ref. 33, as an example, introduce a cooperative, and fully distributed approach to adaptively tune the transmission power of sensors in order to match local connectivity constraints and adaptively maintain the desired neighbor degree on each node. Other solutions (such as Refs. 45 and 46), instead, specify low and high bounds for node degree by defining the local connectivity target as an interval of feasible degrees, i.e., requiring that the degree $\mathrm{K}$ of each node fulfills the condition $\mathrm{Kmin} \leq \mathrm{K} \leq \mathrm{Kmax}$. In Ref. 47 , the authors introduce a mechanism based on explicit notification of non-connected nodes.

\section{Cooperation for prolonging network lifetime}

One of the most important design objectives in wireless sensor networks is to maximize network lifetime by decreasing the energy consumption of sensor nodes as much as possible without sacrificing the necessary Quality-of-Service (QoS) level. All approaches we presented in this paper aim at reducing energy consumption and consequently prolong the network lifetime. In this section, we consider the problem of cooperation for packet forwarding in multi-domain wireless sensor networks.

For the time being it is typical to assume that in a given location there is a single sensor network governed by a single authority. However as the technology for sensor networks gets more mature and standardized, we can expect the co-existence of multiple sensor networks making it possible for sensor nodes of a network to save transmission power spent in forwarding packets of nodes belonging to a different authority. The problem of cooperative packet forwarding in multi-domain sensor networks has not been studied extensively yet. However, we can identify some studies that proved that cooperation in packet forwarding is crucial to ensure operability. Most of them have searched for conditions of cooperation between different domains using game theory as a tool or try to figure out whether cooperation is possible in a multi-domain wireless sensor network without introducing external incentives. Game theory is a theory of decision making under conditions of uncertainty and interdependence. An interesting survey on this topic can be found in Ref. 48.

Authors in Ref. 49 modeled the cooperation in a multi-domain sensor network as a game played by different domains. The objective of players is to maximize their payoff which is defined as the network lifetime of that domain. The authors suggested weight aware strategies by which players choose the next move (decision of whether to request help in forwarding as well as decision of whether to help in forwarding) as a function of previous moves. Using a simulator they searched the strategy space of the game for different topologies aiming to find the equilibrium point which provides the best lifetime values.

In Ref. 50, authors investigate the maximum achievable sensor network lifetime with different multidomain cooperation strategies and in different deployment scenarios through a linear programming framework. Their results show that under certain conditions (sparse network deployment and harsh propagation environment) multi-domain cooperation can extend the wireless network lifetime more than an order of magnitude when compared to non-cooperating domains of wireless sensor networks.

In contrast to the above approaches, authors in Ref. 51 presented a mechanism design that enforces cooperation by punishment. They assume a multi-class (heterogeneous) WSN, where any two non-neighboring class communicate via multi-hop routing. They model the game as that of non-cooperative repeated N-player game between classes of nodes, where nodes participate repeatedly in games with other nodes. In repeated games, a node's action in a given round is influenced by the actions of other nodes and corresponding playoffs in previous rounds. Thus a repeated game offers ways to punish nodes that do not cooperate by decreasing their payoffs at the end of the game. This can be done by tarnished reputation or decrease in incentives resulting in reduced payoffs at the end of the game. Cooperation is similarly rewarded, by examining the payoffs after repeated rounds of the game. Nodes with higher history of collaborative efforts have higher reputation, accumulate incentives faster and are included in reliable routes. By manipulating the parameters (e.g. the amount of gain per forwarding, the designation of charging subject, etc.), those schemes encourage cooperative behavior among selfish nodes. However, as pointed out 
in Ref. 52, if poorly implemented in practice, these incentive schemes themselves have the potential to backfire by offering an incentive to cheat the system in order to gain further benefits.

\section{Conclusion}

In this paper we presented an overview of some of the key areas and research that focus on cooperation in wireless sensor networks. We used examples of recent work to portray the state of art in this area and showed how cooperation among sensor nodes can be exploited at different levels to achieve common goals such as efficient data gathering, accurate event detection and tracking or energy-efficient topology control. One common achievement of cooperation in wireless sensor networks is the reduction of energy consumption and consequently the improvement of the network lifetime.

\section{References}

1. H. Mousannif, H. Al Moatassime and S. Rakrak, An energy-efficient scheme for reporting events over WSNs, International Journal of Pervasive Computing and Communications 7(1) (2011) 44-59. DOI: $10.1108 / 17427371111123685$

2. Q. Wang, M. Hempstead, and W. Yang, A realistic power consumption model for wireless sensor network devices, in IEEE 3rd Annual Communications Society on Sensor and Ad Hoc Communications and Networks (SECON), (Reston, VA, 2006), pp. 286-295. DOI: 10.1109/SAHCN.2006.288433

3. X. Yang, K. Ong, W. Dreschel, K. Zeng, C. Mungle and C. Grimes, Design of a wireless sensor network for longterm, in-situ monitoring of an aqueous environment, Sensors 2(11) (2002) 455-472. DOI:10.3390/s90806411

4. A. Ailamaki, C. Faloutsos, P. Fischbeck, M. Small and J. VanBriesen, An environmental sensor network to determine drinking water quality and security, in ACM SIGMOD Record 32(4) (NY, USA, 2003). DOI: 10.1145/959060.959069

5. A. Mainwaring, J. Polastre, R. Szewczyk, D. Culler and J. Anderson, Wireless sensor networks for habitat monitoring, in Proc. of the 1st ACM international workshop on Wireless sensor networks and applications (NY, USA, 2002), pp. 88-97. DOI: $10.1145 / 570738.570751$

6. S. W. Arms, A. T. Newhard, J. H. Galbreath and C. P. Townsend, Remotely Reprogrammable Wireless Sensor Networks for Structural Health Monitoring Applications, in Proc. of ICCES International Conference on Computational and Experimental Engineering and Sciences (Medeira, Portugal, 2004). DOI:10.1.1.87.9455
7. C. Intanagonwiwat, R. Govindan and D. Estrin, Directed diffusion: a scalable and robust communication paradigm for sensor networks, in Proc. of Mobile Computing and Networking, (ACM, 2000), pp. 56-67 (2000). DOI:10.1.1.117.1253

8. Y. Xue, Y. Cui and K. Nahrstedt, Maximizing lifetime for data aggregation in wireless sensor networks, Mobile Networks and Applications 10(6) (2005) 853-864. DOI: 10.1007/s11036-005-4443-7

9. H. Chen, H. Mineno and T. Mizuno, A meta-data-based data aggregation scheme in clustering wireless sensor networks, in: Proc. of MDM 2006 Workshop MLASN, (2006), pp. 43-49. DOI: 10.1109/MDM.2006.7

10. A. Deligiannakis, Y. Kotidis and N. Roussopoulos, Processing approximate aggregate queries in wireless sensor networks, Information Systems 31(8) (2006) 770 792. DOI:10.1016/j.is.2005.02.001

11. V. Puttagunta and K. Kalpakis, Accuracy vs. lifetime: linear sketches for aggregate queries in sensor networks, Algorithmica 49(4) (2007) 357-385. DOI:10.1.1.1.2775

12. N. Shrivastava, C. Buragohain, D. Agrawal and S. Suri, Medians and beyond: new aggregation techniques for sensor networks, in Proc. of ACM SenSys (2004).DOI: $10.1145 / 1031495.1031524$

13. S. Skeina, The Algorithm Design Manual (SpringerVerlag, New York, 1997), pp. 339-342. DOI: 10.1007/978-1-84800-070-4

14. M. Karpinski and A. Zelikovsky, New Approximation Algorithms for the Steiner Tree Problems, Journal of Combinatorial Optimization 1(1) (1997) 47-65. DOI: 10.1.1.44.9650

15. K. Kalpakis, K. Dasgupta and P. Namjoshi, Efficient algorithms for maximum lifetime data gathering and aggregation in wireless sensor networks, Computer Networks 42(6) (2003) 697-716. DOI: 10.1016/S13891286(03)00212-3

16. K. Dasgupta, K. Kalpakis and P. Namjoshi, Improving the lifetime of sensor networks via intelligent selection of data aggregation trees, in Proc. of the Communication Networks and Distributed Systems Modeling and Simulation Conference (Orlando, Florida, 2003). DOI: 10.1.1.12.5476

17. K. Kalpakis and S. Tang, Collaborative data gathering in wireless sensor networks using measurement cooccurrence, Computer Communications 31(10) (2008) 1979-1992. DOI:10.1016/j.comcom.2008.01.001

18. J. K. Min and C.W. Chung, EDGES: Efficient data gathering in sensor networks using temporal and spatial correlations, The Journal of Systems and Software 83(2) (2010) 271-282. DOI: 10.1016/j.jss.2009.08.004

19. H. Xu, L. Huang, Y. Zhang, H. Huang, S. Jiang and G. Liu, Energy-efficient cooperative data aggregation for wireless sensor networks, Journal of Parallel and Distributed Computing 70(9) (2010) 953-961. DOI: 10.1016/j.jpdc.2010.05.009

20. L. Kai, Z. Wansheng, W. Li and L. Feng, Cooperative Routing for Maximizing Network Performance of 
Wireless Networks. Chinese Journal of Aeronautics 24(2) (2011) 219-223. DOI:10.1016/j.bbr.2011.03.031

21. P. Bonnet, J. Gehrke, and P. Seshadri, Querying the physical world. Personal Communications 7() (2000) 1015. DOI: $10.1109 / 98.878531$

22. B. G. Celler, W. Earnshaw, E. D. Ilsar, L. BetbederMatibet, M. F. Harris, R. Clark, T. Hesketh and N. H. Lovell, Remote monitoring of health status of the elderly at home. A multidisciplinary project on aging at the university of New South Wales, International Journal of Bio-Medical Computing 40(2) (1995) 147-155. DOI:10.1016/0020-7101(95)01139-6

23. H. Song, S. Zhu and G. Cao, Svats: A sensor-networkbased vehicle anti-theft system, in proc. of IEEE INFOCOM, (Phoenix, AZ, 2008), pp. 2128-2136. DOI: 10.1.1.141.6470

24. S. Oh, L. Schenato, P. Chen, and S. Sastry, A scalable real-time multiple-target tracking algorithm for sensor networks, Technical Report UCB//ERL M05/9, (University of California, Berkeley, 2005). DOI: 10.1.1.94.2242

25. V. C. Gungor, O. B. Akan and I. F. Akyildiz, A real-time and reliable transport (rt)2 protocol for wireless sensor and actor networks, IEEE/ACM Transactions on Networking (TON) 16(2) (2008) 359-370. DOI: 10.1109/TNET.2007.900413

26. T. Kieu-Xuan and I. Koo, A collaborative event detection scheme using fuzzy logic in clustered wireless sensor networks, International Journal of Electronics and Communications 65(5) (2011) 485-488. DOI:10.1016/j.aeue.2010.05.002

27. K. Shih, S. Wang, H. Chen and P. Yang, CollECT: collaborative event detection and tracking in wireless heterogeneous sensor networks, Computer Communications 31(14) (2008) 3124-36. DOI: 10.1109/ISCC.2006.50

28. F. Bouabdallah and N. Bouabdallah, The tradeoff between maximizing the sensor network lifetime and the fastest way to report reliably an event using reporting nodes' selection, Computer Communications 31(9) (2008) 1763-1776. DOI: 10.1016/j.comcom.2007.11.020

29. P. Santi, Topology control in wireless ad hoc and sensor networks, ACM Computing Surveys 37(2) (2005) 166194. DOI: $10.1145 / 1089733.1089736$

30. A. Warrier, S. Park, J. Min and I. Rhee, How much energy saving does topology control offer for wireless sensor networks? - A practical study, Computer Communications 31 (14-15) (2008) 1979-1992. DOI: 10.1016/j.comcom.2007.05.019

31. L. Bao and J.J. Garcia-Luna-Aceves, Topology Management in Ad Hoc Networks, in: Proceedings of the 4th ACM international symposium on Mobile ad hoc networking \& computing (MobiHoc'03) (NY, USA, 2003). DOI: $10.1145 / 778415.778432$

32. B. Deb, S. Bhatnagar and B. Nath, STREAM: Sensor Topology Retrieval at Multiple Resolutions,
Telecommunication Systems 26(1) (2004) 285-320. DOI: 10.1.1.7.8570

33. P. Costa, M. Cesana, S. Brambilla and L. Casartelli, A cooperative approach for topology control in Wireless Sensor Networks, Pervasive and Mobile Computing 5(5) (2009) 526-541. DOI: 10.1016/j.pmcj.2009.05.001

34. D.M. Blough, M. Leoncini, G. Resta and P. Santi, The kNeighbors Approach to Interference Bounded and Symmetric Topology Control in Ad Hoc Networks, in: proc. of IEEE Transaction on Mobile Computing 5(9) (2006) 1267-1282. DOI: 10.1109/TMC.2006.139

35. I. Khalil, R. Kronsteiner and G. Kotsis, A semantic solution for data integration in mixed sensor networks, Computer Communications 28(13) (2005) 1564-1574 . DOI: $10.1016 /$ j.comcom.2004.12.041

36. A. Abbasi and M. Younis, A survey on clustering algorithms for wireless sensor networks, Computer Communications 30(14-15) (2007) 2826-2841. DOI: 10.1016/j.comcom.2007.05.024

37. W.R. Heinzelman, A.P. Chandrakasan and H. Balakrishnan, An application-specific protocol architecture for wireless microsensor networks, IEEE Transactions on Wireless Communications 1(4) (2002) 660-670. DOI: 10.1109/TWC.2002.804190

38. S. Lindsey and C.S. Raghavenda, PEGASIS: power efficient gathering in sensor information systems, in Proc. of the IEEE Aerospace Conference (Los Angelos, CA, 2002), pp. 1125-1130. DOI: 10.1109/AERO.2002.1035242

39. O. Younis and S. Fahmy, HEED: A hybrid, energyefficient, distributed clustering approach for ad hoc sensor networks, IEEE Transactions on Mobile Computing 3(4) (2004) 660-669. DOI: 10.1.1.81.2738

40. M. Younis, M. Youssef and K. Arisha, Energy-aware management in cluster-based sensor networks, Computer Networks 43(5) (2003) 649-668. DOI: 10.1016/S13891286(03)00305-0

41. S. Lin, J. Zhang, G. Zhou, L. Gu, J. Stankovic and T. He, ATPC: Adaptive transmission power control for wireless sensor networks, in Proc. of the 4th ACM Int. Conference On Embedded Networked Sensor Systems (SenSys) (ACM, NY, USA, 2006), pp. 223-236. DOI: 10.1145/1182807.1182830

42. C.C. Shen, C. Srisathapornphat, R. Liu, Z. Huang and C. Jaikaeo, CLTC: A cluster-based topology control framework for ad hoc networks, IEEE Transactions on Mobile Computing 3(1) (2004) 18-32. DOI: 10.1109/TMC.2004.1261814

43. R. Wattenhofer, L. Li, P. Bahl and Y.M. Wang, Distributed topology control for power efficient operation in multihop wireless ad hoc networks, in: Proc. of the 20th IEEE INFOCOM (Anchorage, AK, USA, 2001), pp. 1388-1397. DOI: 0.1109/INFCOM.2001.916634

44. C. Yu, K. Shin and B. Lee, Power-stepped protocol: Enhancing spatial utilization in a clustered mobile ad hoc network, Journal on Selected Areas in Communications 

22(7)
(2004)
$1322-1334$.
DOI:

10.1109/JSAC.2004.829349

45. M. Kubisch, H. Karl, A. Wolisz, L. Zhong and J. Rabaey, Distributed algorithms for transmission power control in wireless sensor networks, in: Proc. of IEEE Wireless Communication and Networking Conference WCNC (New Orleans, LA, USA, 2003), pp. 558-563. DOI: 10.1109/WCNC.2003.1200410

46. R. Ramanathan and R. Rosales-Hain, Topology control of multihop wireless networks using transmit power adjustment, in: Proc. of the 19th IEEE INFOCOM Conference (Tel Aviv, Israel, 2000), pp. 404-413. DOI: 10.1109/INFCOM.2000.832213

47. M. Gerharz, C. deWaal, P. Martini and P. James, A cooperative nearest neighbours topology control algorithm for wireless ad hoc networks, in Telecommunication Systems 28(3-4) (2005) 317-331. DOI:10.1007/s11235-004-5575-y

48. R. Machado and S. Tekinay, A survey of game-theoretic approaches in wireless sensor networks, Computer Networks 52(16) (2008) 3047-3061. DOI: 10.1016/j.gaceta.2008.07.003

49. L. Buttyán, T. Holczer and P. Schaffer, Spontaneous cooperation in multidomain sensor networks, Lecture notes in computer science 3813 (2005) 42-53. DOI: 10.1007/11601494_4

50. K. Bicakci and B. Tavli, Prolonging network lifetime with multi-domain cooperation strategies in wireless sensor networks, Ad Hoc Networks 8(6) (2010) 582-596. DOI: 10.1016/j.adhoc.2009.11.004

51. G.V. Crosby and N. Pissinou, Evolution of cooperation in multi-class wireless sensor networks, in Proc. of the 32nd IEEE Conference on Local Computer Networks (IEEE Computer Society Washington, DC, USA, 2007), pp. 489-495. DOI:10.1109/LCN.2007.68

52. E. Huang, J. Crowcroft and I. Wassell, Rethinking incentives for mobile ad hoc networks, in: proc. of ACM SIGCOMM Workshop on Practice and Theory of Incentives in Networked Systems (ACM, NY, USA, 2004), pp. 191-196. DOI: 10.1145/1016527.1016532 